\title{
A Novel Fully Integrated 4-Bit IFM Subsystem Using Band-Stop Filters
}

\author{
S. R. O de Souza ${ }^{1}$ (D) M. F. A de Souza ${ }^{1}$ (D) L. P. Pontes ${ }^{1}$ (D), M. T. de Melo ${ }^{1}$ \\ and Ignacio Llamas-Garro ${ }^{2}$ (C) \\ ${ }^{l}$ Departamento de Eletronica e Sistemas, Universidade Federal de Pernambuco, Recife, Brazil, \\ sromerosouza@gmail.com,marciofbi@gmail.com,leonppontes@gmail.com, marcostdemelo@gmail.com, \\ ${ }^{2}$ Centre Tecnologic de Telecomunicacions de Catalunya (CTTC/CERCA), 08860 Castelldefels, Spain, \\ llamas.ignacio@gmail.com
}

\begin{abstract}
In this paper a 2-4 GHz microstrip IFM (Instantaneous Frequency Measurement) subsystem that uses Wilkinson power dividers, frequency discriminators based on band-stop filters and a DC arduino section is reported. The device uses band-stop filters as an alternative to delay lines, commonly used for IFM. The resonators are responsible for frequency adjustment, according to half guided wavelength resonator length, and the distance between the transmission line and the resonator. A fully micro-controlled $125 \mathrm{MHz}$ resolution IFM subsystem is designed and tested. Results of the IFM subsystem composed by filters, 4-way power dividers and an Arduino board are described.
\end{abstract}

Index Terms - Arduino, Filters, IFM, Microstrip.

\section{INTRODUCTION}

In the last six decades, IFM receivers have been used for monitoring broadband signals of electronic support measures (ESM) systems in the air, sea and land and are widely used in electronic warfare (EW) [1], [2], [4]-[7]. Conventional frequency discriminators compare different time delay intervals with a reference time delay to measure the instantaneous frequency of unknown signals. With this method, the IFM resolution is proportional to the length of the delay lines, related to the frequency discrimination subsystem main elements by bit. The use of open-loop resonators to produce multiband-stop filters is an alternative solution to the use of delay lines, avoiding the reflections and couplings through the meanders and bends commonly used with delay lines to occupy less space [2], [3], [7]. The use of band-stop filters provides more control over the system resolution and further decreases the required simulation time of the whole structure, and additionally provides an output signal similar to the one produced by the use of delay line based interferometers [8]. Multiband-stop filters can be produced by coupling multiple open-loop resonators to a transmission line. The compact multiband-stop filters are ideal to substitute interferometers based on delay lines, traditionally used in IFM subsystems [1], [8], which are bulky and heavy.

The focus of previous work is the design and implementation of multiband-stop filters to replace delay line based single interferometers [2], [3], [7]-[9]. This work includes the design and implementation of a fully integrated IFM subsystem based on [3], in this work an added DC section, where RF to DC converters are used in conjunction with an Arduino board for data processing is 
reported. On the Arduino board, a developed code is used to indicate the sub-band where the unknown input signal falls. The result is sent via USB port to a PC where the result is displayed, indicating the frequency span of the sub-band with its assigned sub-band letter, i.e. A to P. The 16 sub-bands cover the frequency range from 2 to $4 \mathrm{GHz}$.

The automatic result indication provided by the DC section in this work represents a feature that's not used in previous works [2]-[9].

\section{THEORETICAL BACKGROUND}

\section{A. IFM Subsystem Basic Concepts}

Figure 1 shows an IFM subsystem using delay lines to implement four interferometers that define the frequency discrimination channels.

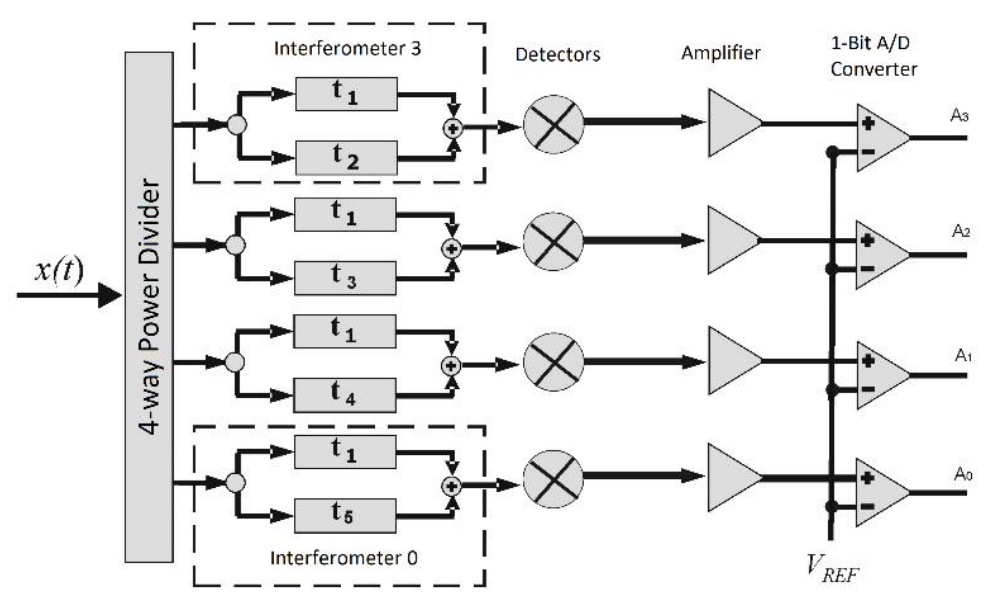

Fig. 1. Topology of an instantaneous frequency measurement subsystem using delay lines.

Figure 2 shows the multiband-stop filters based IFM subsystem. The advantage of using the multiband-stop architecture is related to having only one multiband-stop filter per bit, in place of the delay lines and power dividers used in traditional IFM based on delay line interferometry [9], [10].

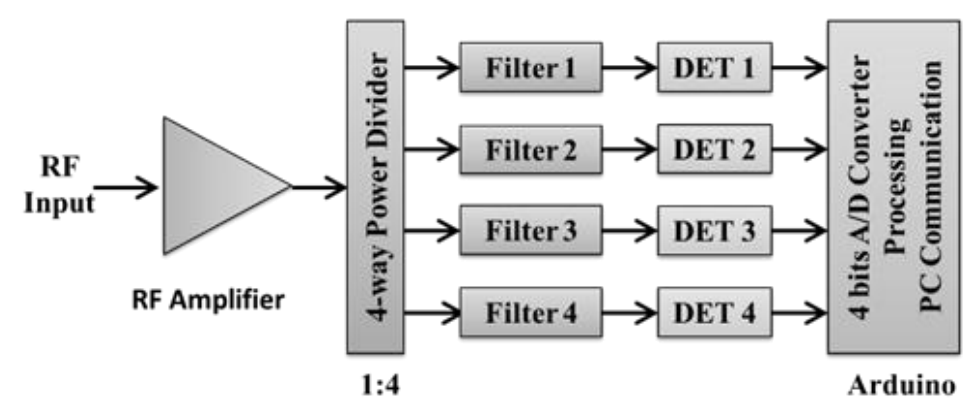

Fig. 2. Topology of an instantaneous frequency measurement subsystem using multiband-stop filters.

In order to achieve a one-step binary code, the response of each multiband-stop filter should resemble the filter responses shown in Fig. 3. 


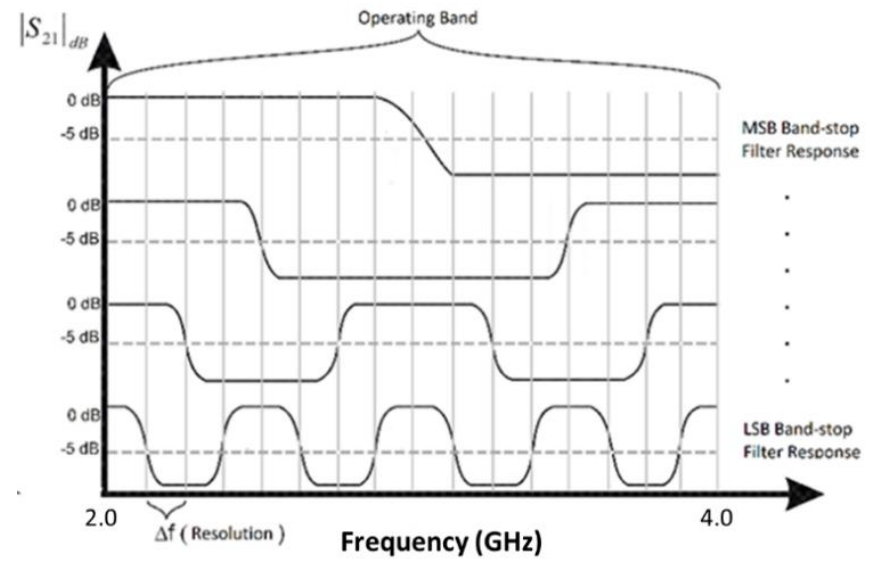

Fig. 3. Ideal multiband-stop filter response.

\section{B. Open-loop resonator}

To design the filters of a four bit IFM subsystem, microstrip based rectangular open loop resonators are used. These resonators have a wide pass-band response and a narrow rejection band [11]. To obtain the desired frequency response for each filter, the resonators are placed close to the $50 \Omega$ transmission line to create the coupling between the resonators and the transmission line. It is important to take into account the coupling between resonators [3,9], to produce an accurate circuit response.

Using the method discussed in this section, the IFM subsystem was designed using open-loop resonators to create four filters, using three Wilkinson power dividers, four RF to DC converters and an Arduino board.

\section{4-BIT IFM SUBSYSTEM}

\section{A. Band-stop Filters Using Open-Loop Resonators}

To design all filters on a same board, the AD1000 substrate is used [3], [12], with dielectric constant $\varepsilon r=10.2 \pm 0.35$, dielectric thickness of $1.27 \mathrm{~mm}$, loss tangent of 0.0023 with copper metallization thickness of $0.035 \mathrm{~mm}$ on both sides. The transmission line thickness that forms all resonators is set to $0.5 \mathrm{~mm}$, and the resonators were all placed at a coupling distance from the main transmission line of $d=0.1 \mathrm{~mm}$. The microstrip transmission line width, is made equal to $1.2 \mathrm{~mm}$ for the main transmission line, having a characteristic impedance of $Z 0=50 \mathrm{ohms}$. The length of the transmission line sections coupled to the main transmission line adjust the resonant frequency of the resonators. The length must be half the wavelength [13] at resonance. The IFM Subsystem operating band is 2 to $4 \mathrm{GHz}$, and the resolution is equal to $125 \mathrm{MHz}$, calculated by dividing the bandwidth by $2^{\wedge} \mathrm{n}$, where $\mathrm{n}$ is the number of filters.

The developed filters for IFM are shown in Fig. 4. All details of the design can be found in [10]. Each group creates a band-stop response. The final geometries were achieved by adjusting and optimizing resonator position along the main transmission line for each filter. To simulate and define 
the desired rejection bands, CST Microwave Studio software was used.
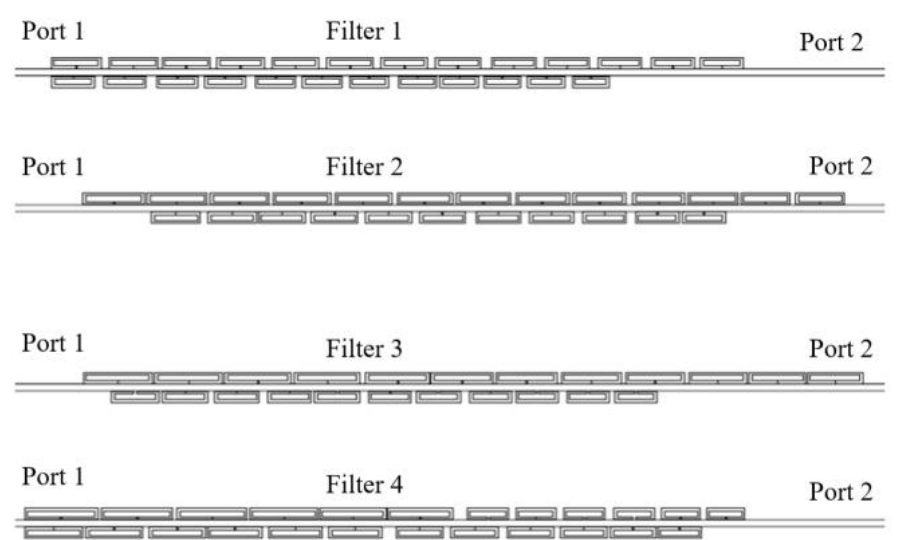

Fig. 4. Band-stop filters for IFM: (a) filter 1 (b) filter 2 (c) filter 3 and (d) filter 4

\section{B. Power Divider}

The 1:4 power divider was designed using three Wilkinson power dividers, with two sections being used to achieve a broad-band response. As each power divider delivers a $-3 \mathrm{~dB}$ output, the complete power divider delivers a quarter of the input signal at each output $(-6 \mathrm{~dB})$. The Wilkinson power divider was chosen because of its reduced dissipation loss compared to others existing topologies, its balanced outputs, which are capable of delivering output signals with the same phase, and their high insulation between the outputs [13]. The layout geometry for the power divider is shown in Fig. 5. In this design $\mathrm{R} 1=100 \mathrm{ohms}$ and $\mathrm{R} 2=200 \mathrm{ohms}$.

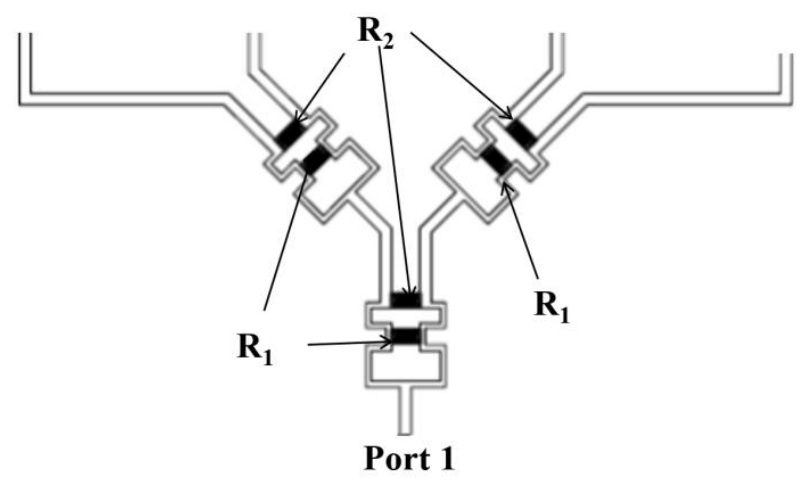

Fig. 5. Power Divider Section

\section{DC Section}

The DC section of the circuit is used to process the data received from the RF section, in order to determine the sub-band where the unknown input signal falls into. The RF detector used is ADL5501 from Analogue Devices. The ADL5501 is a true RMS response power detector device used to convert RF power into a continuous signal, and can operate between $50 \mathrm{MHz}$ and $6 \mathrm{GHz}$. Each ADL5501 delivers a DC level proportional to the RF input to an ADC (Analog-Digital Converter), located at the Arduino board.

The definition of the sub-band of the input signal is done through an Arduino Nano, which is an 
Journal of Microwaves, Optoelectronics and Electromagnetic Applications, Vol. 19, No. 3, September 2020 DOI: http://dx.doi.org/10.1590/2179-10742020v19i3843

electronic hardware prototyping platform that uses an Atmel AVR microcontroller. The Arduino Nano is composed of a single board with embedded data input and output support. The use of an Arduino facilitates the creation of the sub-band finding code of the input signal because the standard programming language used for programming in Arduino is a variation of the $\mathrm{C} / \mathrm{C}++$ language, being essentially the same, with some modifications and proprietary functions proper to the reality of the hardware provided.

The IDE used for developing the code was Arduino IDE 1.8.2. In it, not only the compilation of the code was carried out, but also the programming of the microcontroller and the evaluation of the output values through the serial monitor, responsible for monitoring the values sent via serial by Arduino, making it possible to use this platform as a display and data analysis environment.

The software implemented on the Arduino reads the DC output of each RF detector, computes a ten samples average and after calculates each filter moving average. In Arduino, this value is then compared to the threshold of that bit (each bit has its own threshold level). If the average of a discriminator is below the threshold level, the bit of the output word associated with that discriminator receives logic level 0 . Otherwise, it receives logic level 1 . In this way, the output binary word is generated and the correct frequency sub-band is found, based on Table I.

Fig. 6 shows the basic connections of the ADL5501 [14]. It has an input impedance RF of $50 \Omega$. The COUT capacitor have a value of $100 \mathrm{nF}$. CF LT R was not used.

A set of three SMD capacitors with encapsulation 1206 from Kemet Electronics were used in each detector. Two of these capacitors have a value of $100 \mathrm{nF}$ and one has a value of $100 \mathrm{pF}$, totaling 12 capacitors in the circuit. An Arduino Nano board is used to collect the analog information from the detectors (ports A4 to A7 / pins 23 to 26) and converts these values into digital information, delivering a 4-bit word to the serial port (via the USB port). The 4-bit word relates to the sub-band where the unknown input frequency falls into.

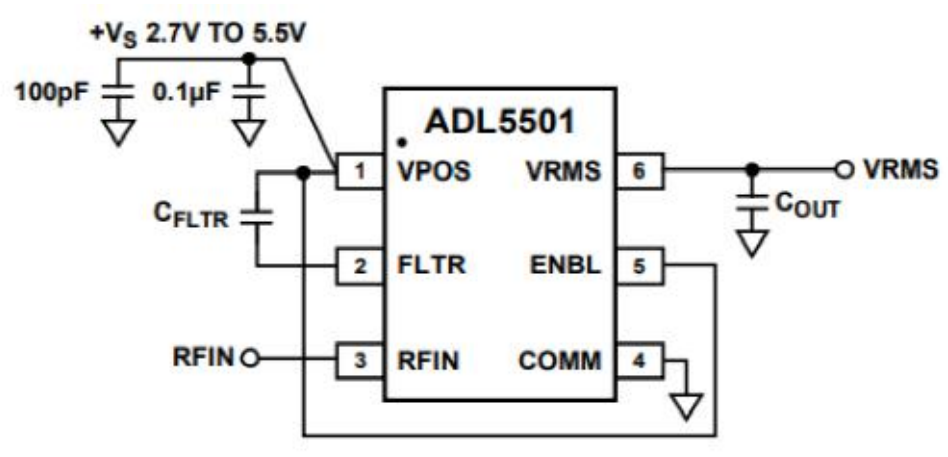

Fig. 6. Basic connection for the ADL5501 [14].

\section{Simulation}

The software CST Microwave Studio was used in this work.

\section{A. Simulated Model and Results}

The device layout model for the IFM Subsystem is shown in Fig. 7. The design is integrated on the 
Journal of Microwaves, Optoelectronics and Electromagnetic Applications, Vol. 19, No. 3, September 2020 DOI: http://dx.doi.org/10.1590/2179-10742020v19i3843

same dielectric substrate, including 4 stop-band filters and the 1: 4 power divider. The 4 RF detectors and the Arduino Nano weren't added to this model, since the goal of this simulation is to find the frequency behavior of the frequency discrimination part of the IFM subsystem only.

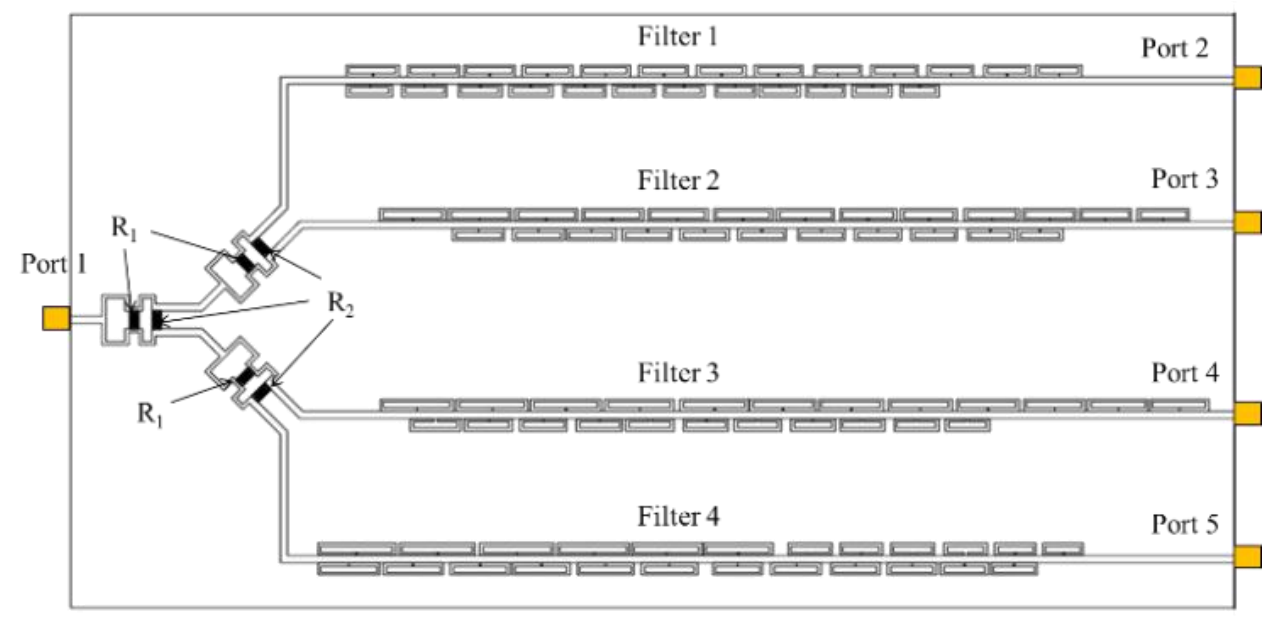

Fig. 7. Simulated model in CST Microwave Studio, where R1 $=100 \mathrm{ohms}$ and R2 $=200 \mathrm{ohms}$.

Fig.8, 9, 10 and 11 show the transmission coefficient results for each multi band-stop filter group, in the bandwidth of operation between 2 and $4 \mathrm{GHz}$.

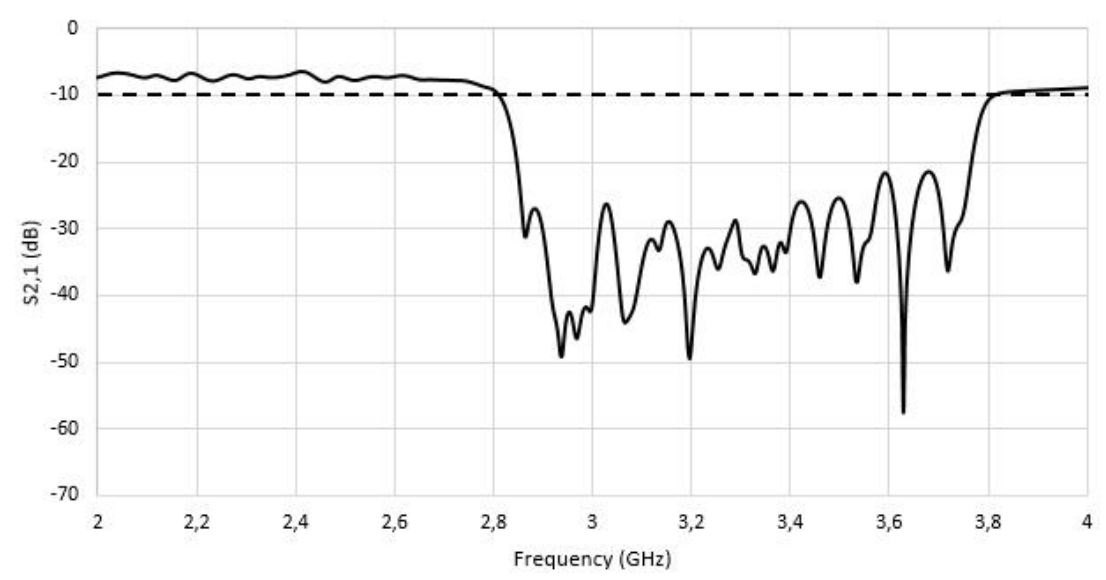

Fig. 8. Simulated transmission coefficient between ports 2 and 1.

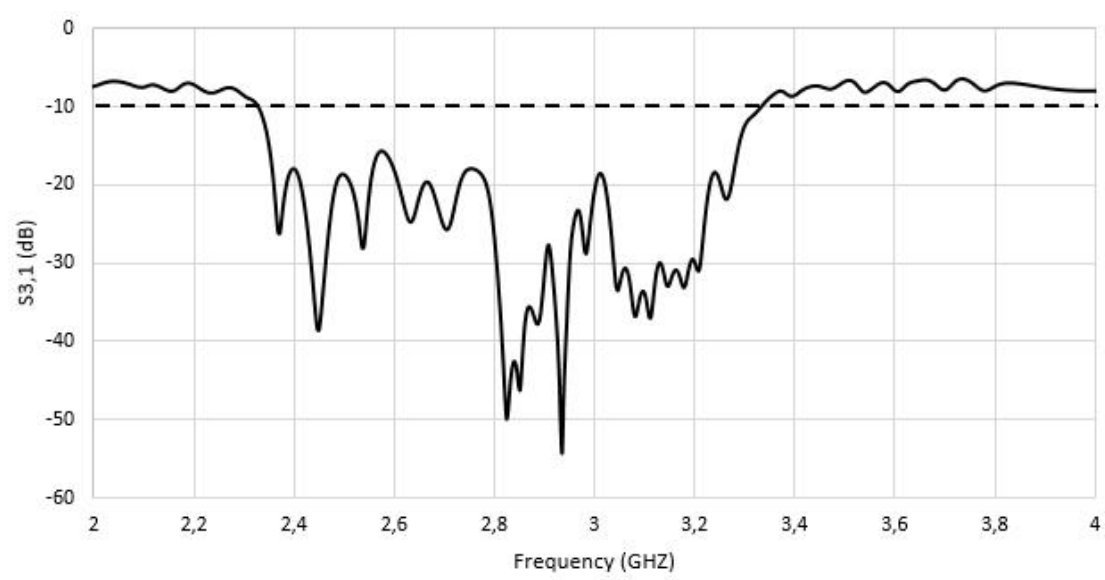

Fig. 9. Simulated transmission coefficient between ports 3 and 1.

Brazilian Microwave and Optoelectronics Society-SBMO received 28 April 2020; for review 1 May 2020; accepted 3 July 2020 


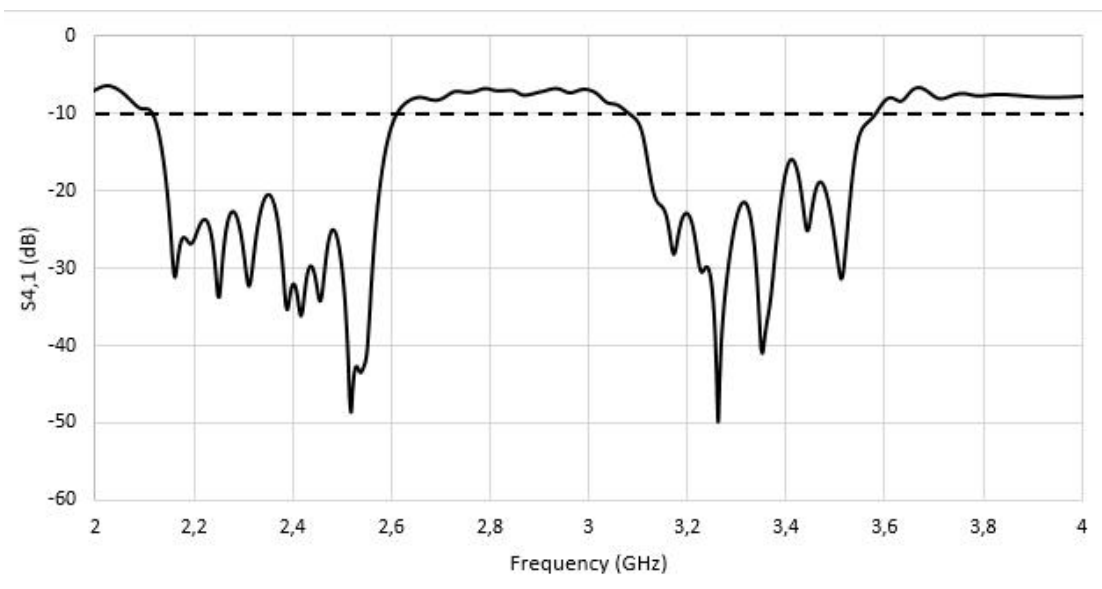

Fig. 10. Simulated transmission coefficient between ports 4 and 1 .

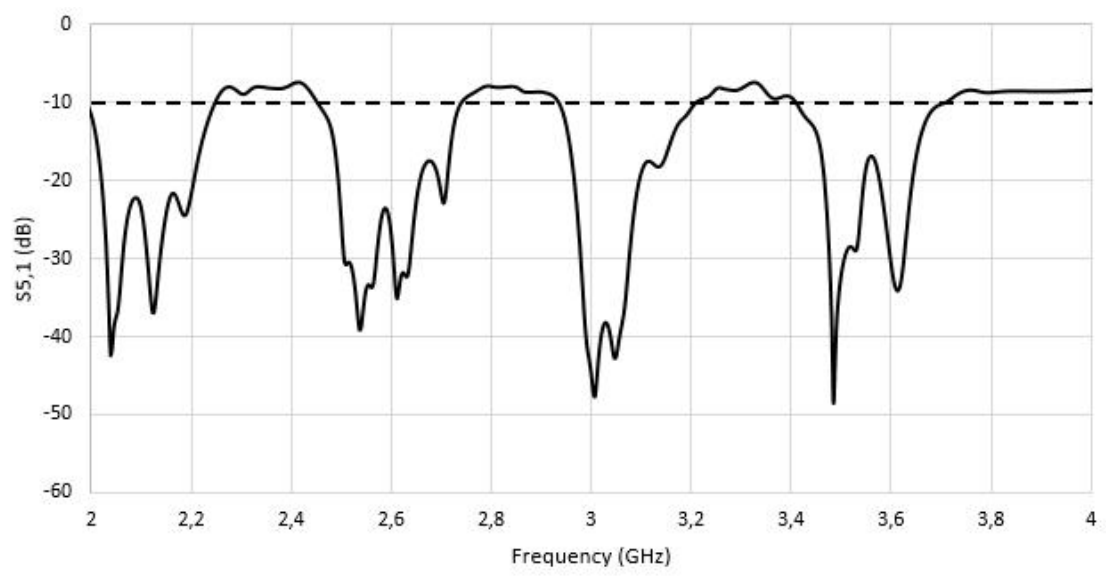

Fig. 11. Simulated transmission coefficient between ports 5 and 1.

These results show a discrepancy when compared to their ideal behavior present in Fig. 3. This shift may be due to some unexpected data cut-off from simulation. This can be avoided, decreasing the size of the cell, which in turn, increases the simulating time as a consequence.

\section{FABRICATION}

\section{A. Fabricated Circuit}

The device was manufactured using a Laser Printed Circuit Board Prototype Machine (LPKF Protolaser S Machine). The fabricated complete IFM subsystem is shown in Fig.12. The characteristic impedance of the input and output ports is $50 \Omega$. The final dimensions of the device are $244.25 \mathrm{~mm} \mathrm{x}$ $99.98 \mathrm{~mm}$, and the smallest line width or spacing is $0.50 \mathrm{~mm}$ IFM. 


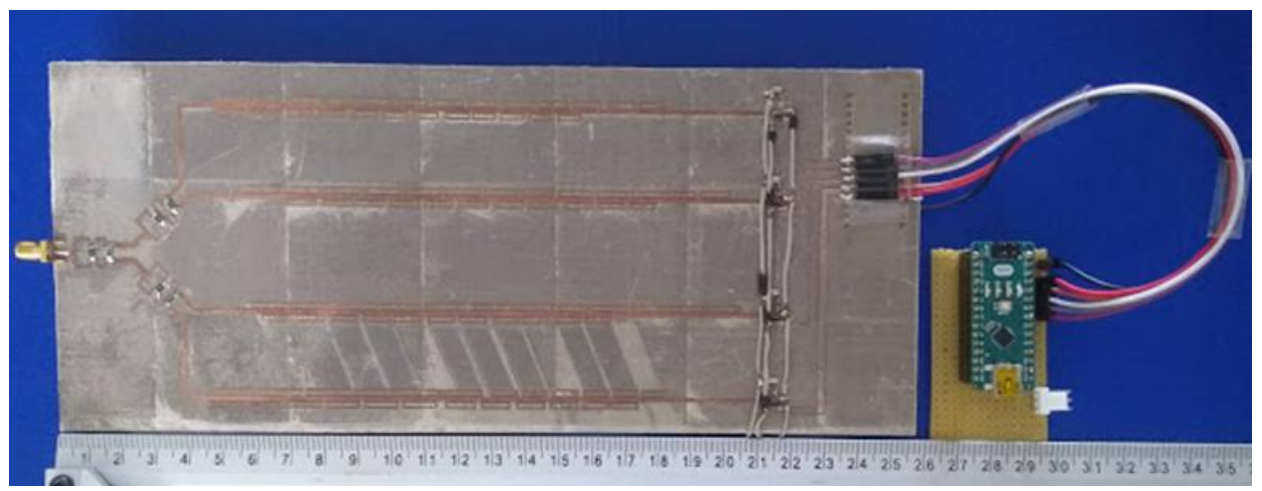

Fig. 12. Fabricated circuit.

\section{B. Measured Results}

After manufacturing the board and soldering the SMA connectors and components for the DC section, the system was tested using an E8257D Analog Signal Generator from Agilent Technologies as an input source, to generate the RF signal to detect, swept in steps through the designed frequency range $(2.0-4.0 \mathrm{GHz})$. After the signal generator, an amplifier from Mini-Circuits, model ZX6083LN-S+ was used to deliver the signal for detection using the proposed IFM subsystem. This amplifier operates between $0.5 \mathrm{GHz}$ and $8.0 \mathrm{GHz}$, with a typical gain of $21 \mathrm{~dB}$ at the IFM system operation frequency range $(2.0-4.0 \mathrm{GHz})$.

Fig. 13 shows the measurement setup. The notebook displayed in the picture was used to read the serial data.

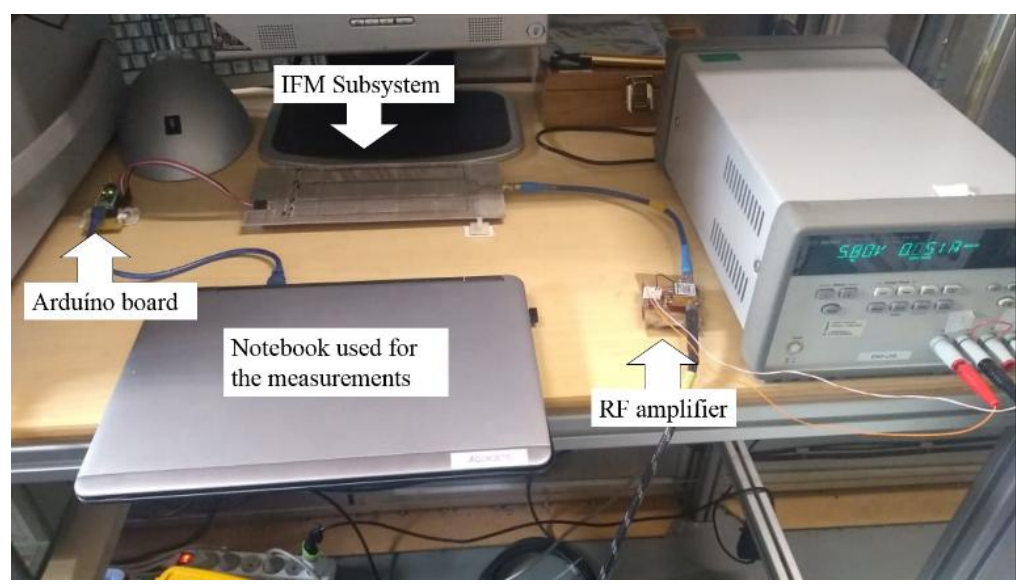

Fig. 13. Measurement Setup. 
TABLE I. MEASURED SUBBANDS

\begin{tabular}{|c|c|c|c|}
\hline $\begin{array}{l}\text { Frequency } \\
\text { (GHz) }\end{array}$ & $\begin{array}{l}\text { Sub- } \\
\text { band }\end{array}$ & $\begin{array}{c}\text { Average Voltage } \\
(\mathrm{mV})\end{array}$ & Converters Voltage (mV) \\
\hline $2.000-2.124$ & 1 & $\geq 330$ and $\leq 500$ & Converter $4>340$ \\
\hline $2.125-2.249$ & 2 & $\geq 330$ & Converter $3>220$ and Converter $4 \leq 330$ \\
\hline $2.250-2.374$ & 3 & $>240$ and $\leq 305$ & Converter $1>340$ and Converter $4<140$ \\
\hline 2.375-2.499 & 4 & $\geq 212$ and $\leq 240$ & Converter $2>300$ \\
\hline $2.500-2.624$ & 5 & $>185$ and $<240$ & Converter $2>90$ and Converter $3<140$ and Converter $4>220$ \\
\hline 2.625-2.749 & 6 & $\geq 167$ and $<210$ & Converter $2<100$ and Converter $3 \geq 133$ \\
\hline $2.750-2.874$ & 7 & $<230$ & Converter $2<120$ and Converter $3>170$ and Converter $4<200$ \\
\hline 2.875-2.999 & 8 & $\geq 250$ & Converter $2 \geq 190$ and Converter $3>350$ and Converter $4<220$ \\
\hline $3.000-3.124$ & 9 & $\geq 240$ and $\leq 400$ & Converter $3 \geq 440$ and Converter $4>400$ \\
\hline $3.125-3.249$ & 10 & $\geq 220$ and $\leq 330$ & Converter $2>140$ and Converter $3<450$ and Converter $4>350$ \\
\hline $3.250-3.374$ & 11 & $\geq 150$ and $\leq 180$ & Converter $1>100$ \\
\hline $3.375-3.499$ & 12 & $\geq 130$ and $\leq 180$ & Converter $1 \leq 97$ \\
\hline $3.500-3.624$ & 13 & $\geq 175$ and $\leq 260$ & Converter $4>220$ and Converter $4<310$ and Converter $3 \neq 175$ \\
\hline 3.625-3.749 & 14 & $\geq 210$ and $\leq 275$ & Converter $2>420$ \\
\hline $3.750-3.874$ & 15 & $\geq 290$ and $\leq 350$ & Converter $2>450$ \\
\hline \multirow[t]{2}{*}{$3.875-4.000$} & 16 & $>360$ and $<400$ & $\begin{array}{l}\text { Converter } 1>260 \text { and Converter } 2>440 \text { and Converter } 3>450 \\
\text { and }\end{array}$ \\
\hline & & & Converter $4>270$ \\
\hline
\end{tabular}

After using the readings from the Arduino board, achieved using the ADL5501, it was possible to identify all 16 different frequency sub-bands. Table 1 shows the criteria used for each sub-band.

Because of the shift in frequency between the ideal frequency response and the obtained in this work, using the average voltages wasn't enough to discern all sub-bands from each other. The voltage readings from the individual converters were used to avoid possible ambiguities. In order to achieve a secure response, the average voltage from the four converters was included in the identification program running on the Arduino board.

For the presented results, the output power of the generator was $-20 \mathrm{dBm}$, meaning that the input power at the board was $1 \mathrm{dBm}$. It is important to point out that the criteria showed at Table I is only valid for this scenario, since with a different input power we would achieve different voltages in the converters inputs.

The minimum and maximum input power level for the converter are $-17 \mathrm{dBm}$ and $+8 \mathrm{dBm}$ [14], respectively. This means that the minimum and maximum input power for the board is close to $11 \mathrm{dBm}$ and $+14 \mathrm{dBm}$, respectively, because of the $6 \mathrm{~dB}$ attenuation caused by the power divider. The dynamic range of operation for the IFM system is $25 \mathrm{~dB}$. 
Fig. 14 shows the serial monitor from the Arduino IDE v.1.8.5, with the sub-band information and individual values of each output converter voltage and the average voltage.

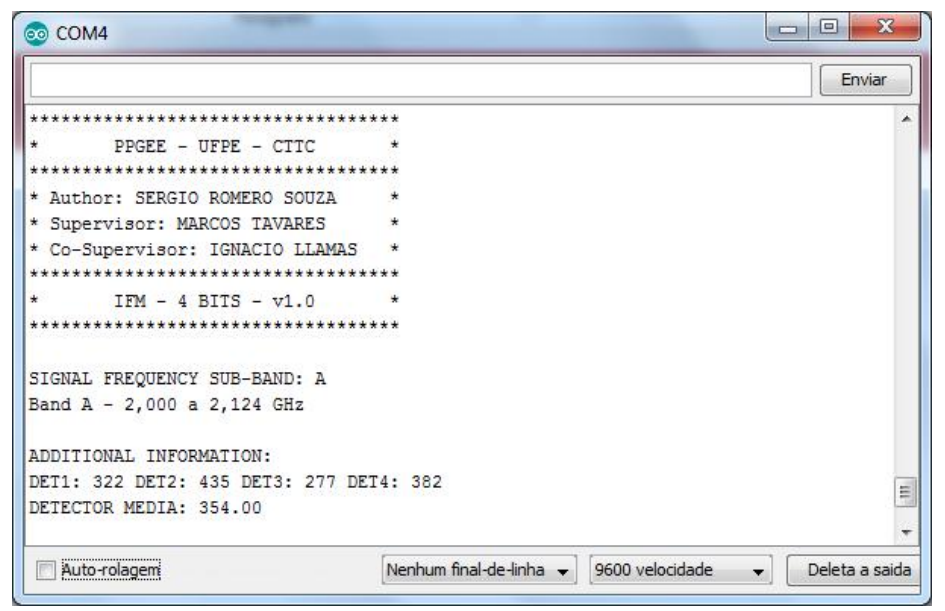

Fig. 14. IFM subsystem display.

\section{CONCLUSION}

The design technique used in the filters allows good control of the resolution and definition of the IFM operating band. In addition, the planar structure enables large-scale commercial production. Integration with arduino allows results to be delivered to the user automatically.

This work can contribute to the development of unknown signal frequency measurement systems, capable of being applied in practice also in locating interfering sources in protected and homologated systems as TV and telecommunication base stations.

\section{ACKNOWLEDGMENT}

This work was financed by FACEPE, CAPES, CNPq and the North Atlantic Treaty Organization, (NATO, G4809), part of this work has been supported by the Generalitat de Catalunya under grant 2017 SGR 891. Thanks to Dr. Fermin Mira at CTTC for fabricating the device.

\section{REFERENCES}

[1] I. Llamas-Garro, de Melo, M. T. and Jung-Mu Kim, Frequency Measurement Technology, Artech House, 2017.

[2] H. Rahimpour, N. Masoumi, "Design and Implementation of a High-Sensitivity and Compact-Size IFM Receiver," IEEE Transactions on Instrumentation and Measurement, vol. 68, no. 7, July 2019.

[3] B. G. M. de Oliveira, M. T. de Melo, I. Llamas-Garro, M.Espinosa, M. R. T de Oliveira, E.M.F de Oliveira, "Integrated Instantaneous Frequency Measurement Subsystem Based on Multi-Band-Stop Filters," Asia Pacific Microwave Conference, APMC, Sendai, Japan, 4-7 November 2014, November 2014.

[4] M. Espinosa, I. Llamas-Garro, B. G. M. de Oliveira, M. T. de Melo, J. M. Kim, "A comparison beetween 4-bit fixed and reconfigurable microwave discriminators for frequency identification," In: The URSI Asia-Pacific Radio Science Conference (URSIAP-RASC), 2016, South Korea. Proceedings of the URSIAsia-Pacific Radio Science Conference (URSI AP-RASC), vol. 1. pp., 2016.

[5] H. Gruchala and M. Czyzewski, "The instantaneous frequency measurement receiver in the complex electromagnetic environment," 15th International Conference on Microwaves Radar and Wireless Communications, vol. 1, 17-19, pp. 155 - 158, MIKON, May 2004.

[6] M, Biehl, A. Vogt, R. Herwig, M. Neuhaus, E. Crocoll, R. Lochschmied,, T. Scherer And W. Jutzi, "A 4 Bit Instantaneous Frequency Meter at $10 \mathrm{Ghz}$ with Coplanar YBCO Delay Line," IEEE Trans. on applied superconductivity, vol. 4, no. 2, pp. 2279-2282, 1995.

[7] M. T. de Melo, M. J. Lancaster and J. S. Hong, "Coplanar Strips Interdigital Delay Line for Instantaneous Frequency Measurement Systems," The Institution of Electrical Engineers, London, Digest, reference number: 1996/226, pp 1/11/4, Nov. 1996. 
Journal of Microwaves, Optoelectronics and Electromagnetic Applications, Vol. 19, No. 3, September 2020 DOI: http://dx.doi.org/10.1590/2179-10742020v19i3843

[8] M. F. A. de Souza, F. R. L e Silva, M. T. de Melo, "A Novel LSB Discriminator for a 5 Bit IFM Subsystem Based on Microstrip BandStop Filter," 38th European Microwave Week Conference Proceedings, pp. 36-39, Amsterdam, 2008

[9] E. M. F. Oliveira, T. L. Pedrosa, B. G. M. de Oliveira, S. R. de Oliveira, M. T. de Melo, I. Llamas-Garro, "A Novel Microstrip Frequency Discriminator for IFM Based on Balanced Gray-code," In: IEEE SBMO IMOC2017, 2017, Aguas de Lindoia. Proceeding of the IEEE SBMO IMOC2017, vol. 1, pp. 1-4, 2017.

[10] M. F. A. de Souza, F. R. L. Silva, M. T. Melo, L. R. G. S. L. Novo, "Discriminators for Instantaneous Frequency Measurement Subsystem Based on Open-Loop Resonators," IEEE Transactions on Microwave Theory and Techniques, vol. 57, pp. 2224-2231, 2009.

[11] A. Gorur, "Bandstop filter with a wider upper passband using microstrip open-loop resonator," Proceeding of APMC2001, Taipei, Taiwan, 2001.

[12] Rogers Corporation (2019) AD1000, AD1000 $\quad$ TM Data Sheet.[Online] Available: https://www.rogerscorp.com/documents/3269/acs/AD1000-Data-Sheet.pdf.

[13] E. S. Azevedo, B. G. M. Oliveira, M. T. Melo, "Microstrip Power Divider for Integration of an Instantaneous Frequency Measurement System," SBMO/IEEE MTT-S International Microwave and Optoelectronics Conference, pp. 295-297, October/November 2011.

[14] Analog Devices (2017) ADL5501 Datasheet and Product Info. [Online] Available: http://www.analog.com/en/products/rf-microwave/rf-power-detectors/rms-responding-power detectors/ad15501.html\#product-overviewRO3010-RO3035.pdf. 